\title{
Inequality, Intergenerational Mobility and Redistributive Policies under Endogenous Information
}

\author{
Tommaso Gabrieli ${ }^{1}$ \\ University of Reading
}

\begin{abstract}
This paper presents a dynamic model to study how different levels of information about the root determinants of wealth (luck versus effort) can impact inequality and intergenerational mobility through societal beliefs, individual choices and redistributive policies. To my knowledge, the model presented is the first dynamic model in which skills are stochastic and both beliefs and voted redistribution are determined endogenously. The model is able to explain a number of empirical facts. Large empirical evidence shows that the difference in the political support for redistribution appears to reflect differences in the social perceptions regarding the determinants of individual wealth and the underlying sources of income inequality. Moreover the beliefs about the determinants of wealth impact individual choices of effort and therefore the beliefs about the determinants of wealth impact inequality and mobility both through choices of effort and redistributive policies. The model generates multiple equilibria (US versus Europe-type) which may account for the observed features not only in terms of societal beliefs and redistribution but also in terms of perceived versus real mobility and inequality.
\end{abstract}

JEL Classification: D31, D72, D80, E62, H30, O40

Keywords: Politico-Economic Equilibria, Redistribution, Inequality, Intergenerational Mobility, Incomplete Information

\section{Introduction}

This paper presents a dynamic model to study how different levels of information about the root determinants of wealth (luck versus effort) can impact inequality and intergenerational mobility through societal beliefs, individual choices and redistributive policies. In a companion paper (Gabrieli (2010)), I analyze how different levels of information about the determinants of individual wealth ${ }^{2}$ can affect, through individual beliefs, the individual preferences over redistribution and the individual optimal decisions of how much effort to exert. As a further step, Gabrieli (2010) analyzes the impact of incomplete information on the prevailing level of redistribution under majority voting and on aggregate outcomes as aggregate effort, aggregate output and welfare. The model of Gabrieli (2010) offers policy results in terms of comparative statics and insights about the various observed differences between laissez-faire versus welfare 
state type of economies, but since it describes a one period economy and does not analyze savings, wealth accumulation or intergenerational transfers, the model cannot say much about the dynamics of inequality and wealth mobility over time. In this paper I take the natural further step to study how incomplete information can impact beliefs, political and economic outcomes in a dynamic set up in order to gain insights about the dynamics of inequality and wealth mobility. Such exercise seems to be important: if individual beliefs about the underlying determinants of wealth are important determinants of individual voting and effort choices, then, through those choices, the same beliefs become important determinants of the dynamics of inequality and mobility. Another reason is that individual beliefs about the determinants of wealth are intrinsically related to individual beliefs about the determinants and the extent of mobility, which is a dynamic process, it is therefore important to study such beliefs in a dynamic setting. In order to gain such insights, the present paper introduces the described set up with varying levels of information in an intergenerational model of bequests with stochastically evolving skills.

The present model is made of three main building blocks. The first element is a dynamic set-up with with bequests and stochastic skills which allows to analyze intergenerational inequality and mobility. The second block is given by the political economy side: a linear redistribution scheme, where the prevailing rate of redistribution is set by the median voter. The third block is the information structure: in every period the level of information about the true value of individual return on effort (skills) can vary in a continuous way from a completely uninformative structure to the case that each individual is perfectly informed about her own skills. The level of information should be interpreted as an institutional feature of the economy which governments or other institutions can possibly affect through various policies: educational policies ${ }^{3}$, the release of information on past mobility ${ }^{4}$ or pure propaganda ${ }^{5}$. The model is used to conduct two main policy exercises. One exercise consists in analyzing how different levels of incomplete information affect the endogenous outcomes (individual voting and effort, redistribution, aggregate effort, output and welfare). Another exercise consists in considering also the level of information as endogenous and analyzing which levels of information are optimal for the society and can arise in an equilibrium. This paper constitutes a first attempt to link three different strands of theoretical literature: models of intergenerational inequality, models on the political economy of redistribution, models which analyze the role of individual and collective beliefs about the underlying determinants of wealth. A short review of the the related literature follows. ${ }^{6}$

(i) Neoclassical models of intergenerational inequality. It is the seminal paper of Stiglitz (1969) to be commonly considered the first modern analysis of the distribution of wealth and income among individuals. The model of Stiglitz presents a strong result of long run convergence in the dynamics of individual income which parallels the seminal result obtained by Solow (1956) in the context of country income. In the model of 
Stiglitz agents are endowed with capital (accumulated factor) and labor (non accumulated factor), markets are competitive and both factors are paid at their marginal return. The assumptions of diminishing returns to capital and of an identical concave saving function across individuals imply that individual wealth increases over time in a concave fashion and eventually converges to a steady state value which does not depend on the initial level of wealth. In other words, in the model inequality across families is solely determined by the differences in the non accumulated factors (i.e. the differences in individual skills) and when all families are equally endowed with the non accumulated factor (i.e. skills are homogenous across families) every family converges to the same level of wealth. Building on this seminal model, other authors have extended the basic set-up in order to study intergenerational inequality and mobility. In this context, saving choices have been micro-founded as decisions to leave bequests for the future generation. For what concerns bequests, two main formalization have been used in the literature: one in which bequests enter directly into the utility function of the parents (Atkinson (1980)) and one in which parents care about their children's utility per se (Becker and Tomes (1979)). Those two alternative formulations can give different conclusions about the dynamics of accumulation, inequality and the effects of redistributive taxation. Developing this type of analysis, Becker and Tomes (1986) focus on the intergenerational transmission of abilities across generations and study the implications for the investments in human capital and the resulting dynamics of inequality. ${ }^{7}$

More recently there has been extensive work on models with credit market imperfections and poverty traps. The basic idea behind the effect of imperfect financial markets is that if poor individuals are prevented from borrowing and hence cannot invest, then in a dynamic context initial inequalities may persist and some dynasties remain stuck into a poverty trap. Therefore these models can produce persistent inequality across dynasties abstracting from the effect of skills' differences. Relevant papers in this group include those of Galor and Zeira (1993), Banerjee and Newman (1993), Piketty (1997), Aghion and Bolton (1997), among others.

(ii) Models of political economy. The contribution of these models is represented by the fact that the prevailing level of redistribution is not exogenous but it is the result of a voting process. This idea has been introduced by the seminal paper of Meltzer and Richard (1981) and the seminal contributions which introduced it in dynamic macroeconomic models are those of Perotti (1993), Alesina and Rodrik (1994) and Persson and Tabellini (1994). The main idea behind those models is that, as in Meltzer and Richard (1981), given the median voter theorem, greater inequality translates into a poorer median voter relative to the country's mean income and therefore the greater the inequality and the higher it is the voted level of redistribution in the economy. High levels of redistribution in turn lower individual incentives to accumulate capital and hence the result that inequality lowers growth. In those models inequality derives from the fact that skills are fixed and persistently different across dynasties. Therefore 
such models are focused on the study of the determinants and the implications of redistribution but cannot give insights about the dynamics of mobility. Such models have been quite influential, especially in bringing endogenous political choices into the big picture. They also stimulated a great deal of discussion about the relationship between inequality, growth and redistribution. Some empirical evidence challenged the conclusion on the basis of two different observations. ${ }^{8}$ The first observation is that it does not always seem to be the case that inequality is detrimental to growth, even though the evidence in favor is quite large. The second and major challenge comes from the observation that it does not seem to be the case that more inequality implies higher redistribution. This second challenge inspired a new group of theoretical models whose major focus is to explain the described evidence relating inequality and redistributive politics. These models achieve this result showing the existence of multiple equilibria: a Europe type equilibrium characterized by relatively lower inequality and higher redistribution versus a US type equilibrium characterized by relatively higher inequality and lower redistribution. In the model of Benabou (2000) the prevailing level of redistribution is still a voting outcome, but unlike in the previously mentioned models the relationship between inequality and redistribution is not monotonic. Other theoretical models which obtain multiple equilibria with similar features are those of Saint-Paul (2001) and Hassler, Rodriguez-Mora, Storesletten, and Zilibotti (2003). Despite the fact that in the model of Benabou (2000) skills evolve stochastically across generations, every generation exerts effort before knowing the realization of ability and because of the same prior on the value of abilities, at each period all individuals exert the same value of effort. Therefore in the model there are no dynasties which remain stuck in poverty and the model cannot give insights about the dynamics of mobility.

(iii) Models which focus on the role of beliefs. Starting from the evidence that the beliefs held by people about the underlying determinants of individual wealth and social mobility appear to be strong determinants of voted redistribution, the theoretical contributions of Piketty (1995), Alesina and Angeletos (2005) and Benabou and Tirole (2006) have developed insightful models describing how individual beliefs can shape politico-economic outcomes and viceversa and how multiple equilibria (US-type vs Europe-type) are possible. These models with beliefs can explain how beliefs affect redistribution and effort choices in static set-ups, but not how beliefs can affect intergenerational inequality and mobility. ${ }^{9}$

In my model the amount of wealth left as bequest enter in the utility function of the parents as in the models of Atkinson (1980) and Alesina and Angeletos (2005). This is a convenient formalization which simplifies the dynamic problem to a great extent because it implies that every generation only wants to maximize present wealth and avoids issues of inter-temporal optimization and dynamic voting. The aim of this paper is to build a dynamic model with bequests, stochastic skills, endogenous voting and endogenous information. Stochastic skills are an important ingredient to study mobility and to allow for incomplete information about the determinants of wealth but 
imply some technical issues which will be discussed. Given such technical issues, it is convenient to build the full model by steps, adding each building block separately. After introducing the general set-up in section 2, section 3 analyzes the case of exogenous political outcome and exogenous information, section 4 introduces voting but maintains exogenous information, section 5 allows for voting and endogenous information, the last section draws some final remarks.

\section{Set Up with Bequests}

I consider an economy populated by a continuum of non-overlapping generations $i \in$ $[0,1]$. Each generation (or agent) lives for one period $t$ and is labeled by $i_{t}$. Each generation $i_{t}$ produces output $y_{t}^{i}$ with the following technology:

$$
y_{t}^{i}=\theta_{t}^{i} e_{t}^{i}+k_{t-1}^{i},
$$

where $k_{t-1}^{i}$ represents the bequest or other parental investment received by the previous generation, $e_{t}^{i}$ is the effort implemented by generation $i_{t}$ and $\theta_{t}^{i}$ is the return to effort or productivity. With respect to abilities, I consider both the case in which abilities are i.i.d across different dynasties $i$ but persistent over the life of a dynasty $\left(\theta_{t}^{i}=\theta^{i}\right)$ and the more interesting case that $\theta_{t}^{i}$ is random and i.i.d. across $i$ and $t$. As standard in this literature, agents face the linear tax/redistribution scheme introduced by Romer (1975). The individual budget constraint is given by

$$
c_{t}^{i}+k_{t}^{i}=w_{t}^{i}=\left(1-\tau_{t}\right) y_{t}^{i}+\tau_{t} \bar{y}_{t},
$$

where $c_{t}^{i}$ denotes own consumption, $k_{t}^{i}$ is the bequest left to the next generation, $w_{t}^{i}$ denotes disposable wealth, $\tau_{t}$ is the tax rate, $\tau_{t} \bar{y}_{t}$ is the lump-sum transfer and $\bar{y}_{t}$ is the mean output in generation $t$. Each agent votes for the tax rate $\tau_{t}$ and exerts effort after that the tax rate is announced. After that, individuals receive neat wealth according to (2) and decide how much to consume or to leave as bequests out of it. The private utility of each agent is given by the following function:

$$
u_{t}^{i}\left(c_{t}^{i}, k_{t}^{i}, e_{t}^{i}\right)=\frac{1}{(1-\alpha)^{1-\alpha} \alpha^{\alpha}}\left(c_{t}^{i}\right)^{1-\alpha}\left(k_{t}^{i}\right)^{\alpha}-\frac{b e_{t}^{i^{2}}}{2} .
$$

The first term in (3) represents the utility from own consumption and bequests, whereas the second term is the negative utility of exerting effort.

Following Alesina and Angeletos (2005) and Atkinson (1980) I assume a CobbDouglas function over consumption and bequests with $\alpha \in(0,1)$, which together with the constant $\frac{1}{(1-\alpha)^{1-\alpha} \alpha^{\alpha}}$ implies that $\alpha \in(0,1)$ denotes the fraction of wealth allocated to bequests, maintaining the dynamic problem very tractable. Agent $i_{t}$ chooses consumption, bequest and effort $\left(c_{t}^{i}, k_{t}^{i}, e_{t}^{i}\right)$ so as to maximize utility subject to the bud- 
get constraint, taking the political outcome $\left(\tau_{t}\right)$ as given. It follows that the optimal individual consumption and bequest are respectively

$$
c_{t}^{i}=(1-\alpha) w_{t}^{i}
$$

and

$$
k_{t}^{i}=\alpha w_{t}^{i} .
$$

The indirect utility function in terms of wealth thus reduces to

$$
u_{t}^{i}=w_{t}^{i}-\frac{b e_{t}^{i^{2}}}{2} .
$$

This set up allows to avoid inter-temporal optimization and problems of dynamic voting.

\section{Step 1: Exogenous Tax Rate and Exogenous Information.}

In this first version of the model I maintain two assumptions: (i) I abstract from voting over $\tau$ considering this political outcome to be exogenously determined and constant over time $\left(\tau_{t}=\tau\right)$, (ii) I consider the case that each individual $i$ is fully informed about the value of $\theta^{i}$. This section shows results which are already known by the previous literature but it is important for building the rest of the analysis.

Plugging the expression for pre-tax wealth (1) into the utility function (6) and solving the f.o.c. I find the expression for the individual optimal effort:

$$
e_{t}^{i}=\left(1-\tau_{t}\right) \theta_{t}^{i} / b
$$

Plugging the expression for optimal effort (7) and the expression for pre-tax wealth (1) into the individual budget constraint (2) I find the law of motion of bequests:

$$
k_{t+1}^{i}=\alpha\left((1-\tau) k_{t}^{i}+(1-\tau)^{2} \theta_{t}^{i^{2}} / b+\tau \bar{k}_{t}+\tau(1-\tau) \bar{\theta}_{t}^{2} / b\right),
$$

which determines also the law of motion of wealth given (5). In the case in which abilities are i.i.d. across different dynasties but constant over the life of a dynasty $i$ $\left(\theta_{t}^{i}=\theta^{i}\right)$, the law of motion (8) describes a convergent auto-regressive process. ${ }^{10}$ It is immediate to derive the steady-state bequest of dynasty $i$

$$
k^{i}=\frac{\alpha\left((1-\tau)^{2} \theta^{i^{2}} / b+\tau(1-\tau) \bar{\theta}^{2} / b+\tau \bar{k}\right)}{1-\alpha(1-\tau)}
$$


and the steady-state mean (or aggregate) bequest

$$
\bar{k}=\frac{\alpha(1-\tau) \bar{\theta}^{2}}{b(1-\alpha)} .
$$

Not surprisingly, given that the only source of heterogeneity is in the abilities $\theta^{i}$, expression (9) shows that the greater is $\theta^{i}$ and the greater is the steady-state wealth. It is also obvious that redistribution has an equalizing effect. From (9) and (10) it can be computed that the difference between mean and individual $i$ bequest equals

$$
\bar{k}-k^{i}=\frac{\alpha(1-\tau)^{2}\left(\bar{\theta}^{2}-\theta^{i^{2}}\right)}{b(1-\alpha(1-\tau))}
$$

and decreases in the tax rate $\tau$. At the same time expression (10) shows that redistribution diminishes aggregate bequest and hence aggregate wealth. This trade-off between redistribution and growth is due to the fact that individual effort (7) decreases in the tax rate and redistribution has no other effect on output ${ }^{11}$.

In order to have some more insights about the intergenerational dynamics of inequality and mobility it is interesting to explore the case that abilities are not persistent over the life of a dynasty. Assuming that abilities are drawn at random for each generation $t$ and that $\theta_{t}^{i^{2}}=\bar{\theta}^{2}+\varepsilon_{t}^{i}$, where $\varepsilon_{t}^{i}$ is an i.i.d. error term across $i$ and $t$, with 0 mean, variance equal to $\sigma^{2}$ and zero serial correlation, the law of motion (8) still describes a convergent auto-regressive process. ${ }^{12}$ The steady state mean bequest is still given by expression (10) and the variance is equal to

$$
\operatorname{var}\left(k^{i}\right)=\frac{\alpha^{2}(1-\tau)^{4} \sigma^{2}}{b^{2}\left(1-\alpha^{2}(1-\tau)^{2}\right)} .
$$

As in the case with persistent abilities, increasing the rate of redistribution reduces inequality across agents. 13

This model predicts convergency to a steady state value of wealth which does not depend on the initial level of wealth. The version with persistent abilities is qualitatively very similar to the seminal model of Stiglitz (1969) which is widely known for a Solow-type convergency in the context of individual wealth. Stiglitz (1969) model shows that with a concave saving function - in this case bequest function - if abilities are identical for everybody, everybody will converge to the same wealth, regardless of the amount of initial wealth. With heterogenous abilities, inequality is driven by abilities but once again initial wealth inequality does not matter in the long run. ${ }^{14}$ Plugging the expression of optimal effort (7) and the expression of pre-tax wealth (1) into the utility function (6) gives the expression for expected utility as a function of the tax rate $\tau$ :

$$
\bar{u}=\bar{y}-\frac{c \overline{e^{2}}}{2}=\bar{k}+\frac{\left(1-\tau^{2}\right) \bar{\theta}^{2}}{2 b} .
$$


Expected utility is maximized by a zero tax rate. The reason is that the utility function is linear in wealth and therefore there are no ex-ante gains from redistribution; on the other hand effort and output decrease in the tax rate.

\section{Case of Incomplete Information.}

Leading to the analysis presented in the next sections, it is useful to analyze the case in which agents have incomplete information and expect to be of average ability $\bar{\theta}$. In this case the expression for individual optimal effort is

$$
e_{t}^{i}=\left(1-\tau_{t}\right) \bar{\theta} / b .
$$

Plugging this expression and the expression for pre-tax wealth (1) into the individual budget constraint (2) gives the law of motion of bequests:

$$
k_{t+1}^{i}=\alpha\left((1-\tau) k_{t}^{i}+(1-\tau)^{2}(\bar{\theta})^{2} / b+\tau \bar{k}_{t}+\tau(1-\tau)(\bar{\theta})^{2} / b\right) .
$$

The steady-state individual bequest, mean bequest and variance respectively follow:

$$
\begin{gathered}
k^{i}=\frac{\alpha\left((1-\tau)^{2} \bar{\theta} \theta^{i} / b+\tau(1-\tau)(\bar{\theta})^{2} / b+\tau \bar{k}\right)}{1-\alpha(1-\tau)}, \\
\bar{k}=\frac{\alpha(1-\tau)(\bar{\theta})^{2}}{b(1-\alpha)}, \\
\operatorname{var}(k)=\frac{(1-\tau)^{4} \sigma^{2}}{b^{2}\left(1-\alpha^{2}(1-\tau)^{2}\right)} .
\end{gathered}
$$

Given that $\bar{\theta}^{2}>(\bar{\theta})^{2}$, the mean wealth with complete information (10) is strictly greater than the mean wealth under incomplete information (17). ${ }^{15}$ The same beneficial effect of information appears by comparing the expression of expected utility in the case of complete information (13) with the respective expression in the case of incomplete information:

$$
\bar{u}=\bar{k}+\frac{\left(1-\tau^{2}\right)(\bar{\theta})^{2}}{2 b} .
$$

From (16) and (17) it can be computed that under incomplete information the difference between mean and individual bequests equals

$$
\bar{k}-k^{i}=\frac{\alpha(1-\tau)^{2} \bar{\theta}\left(\bar{\theta}-\theta^{i}\right)}{b(1-\alpha(1-\tau))}
$$

and decreases in the tax rate $\tau$.

It is easy to notice that the steady state with incomplete information is characterized by lower inequality than the steady state with incomplete information, as the dif- 
ference between expressions (11) and (20) is equal to $\alpha(1-\tau)^{2}(1-\pi)\left(\theta_{H}-\theta_{L}\right)\left(\pi \theta_{H}+\right.$ $\left.(1-\pi) \theta_{L}\right) b(1-\alpha(1-\tau))$ which is a positive term given $\tau<1$.

\section{Step 2: Endogenous Tax Rate and Exogenous Information.}

The second step of the analysis is to introduce voting. Agents vote for the tax rate before exerting effort; solving backward I find the objective function of voter $i_{t}$ by plugging the expression of optimal effort (7) and the expression for net wealth (2) into the utility function (6) and then maximizing the obtained expression with respect to $\tau$. Each voter $i$ maximizes the following indirect utility function in $\tau$ :

$$
u_{t}^{i}=\tau\left(k_{t}^{i}-\bar{k}\right)+(1-\tau)^{2} \theta^{i^{2}} / b+\tau(1-\tau) \bar{\theta}^{2} / b-(1-\tau)^{2} \theta^{i^{2}} / 2 b .
$$

Assuming for the moment that the second derivative of expression (21) with respect to $\tau$ is strictly negative, the first order condition gives the ideal tax rate of voter $i$ :

$$
\tau_{t}^{i}\left(k_{t}^{i}, \theta^{i}\right)=1-\frac{1+\frac{b\left(k_{t}^{i}-\overline{k_{t}}\right)}{\theta^{2}}}{2-\frac{\theta^{i 2}}{\bar{\theta}^{2}}} .
$$

Both the numerator and the denominator of (22) show that the gains from redistribution are traded off the moral hazard effect of taxation. I introduce the following assumption in order to assure the concavity of the objective function (21) and therefore in order to use the median voter theorem:

Assumption 1: $2 \theta_{L}^{2}>\theta_{H}^{2}$.

A proposition follows:

Proposition 1. The individual preferences for taxation are single-peaked and the individual ideal tax rate is given by expression (22).

Proof. The second derivative of the objective function (21) is given by the following expression:

$$
\frac{d^{2} u_{t}^{i}}{d \tau}=\frac{\left(\theta^{i}\right)^{2}-2 \bar{\theta}^{2}}{b}
$$

The condition stated by assumption 1 is sufficient for (23) to be strictly negative as the maximum value that $\left(\theta^{i}\right)^{2}$ can take is $\theta_{H}^{2}$ and the minimum value that $2 \theta^{2}$ can take is $2 \theta_{L}^{2}$.

Proposition 1 shows that preferences over the tax rate are single peaked and therefore the median voter theorem applies. In order to analyze a steady state of the dynamic model, I look for a steady state such that given a stationary history $\tau_{s}=\tau$ for all generations $s \leq t-1$, then $\tau_{t}=\tau$ is optimal for generation $t$. 


\section{Persistent Abilities across Generations}

I first analyze the case in which abilities are persistent over dynasties, namely $\theta_{t}^{i}=\theta^{i}$. Consider a stationary history $\tau_{s}=\tau$ for all generations $s \leq t-1$. For each dynasty $i$ the low of motion (8) implies that the value of the bequest converges to expression (9) and that the mean value converges to expression (10). Given persistent abilities $\left(\theta_{t}^{i}=\theta^{i}\right)$ and assuming that every dynasty starts life with no endowment $\left(k_{0}^{i}=0\right.$ for all $\left.i\right)$, in every period $t$ the median and prevailing tax rate $\tau_{t}$ is that one of the dynasty with median ability $\theta^{m}$. Plugging the expressions of the steady state median bequest $k^{m}$ (obtained through (9) ) and of the steady state mean bequest (10) into the expression for the individual ideal tax rate (22) and using (11), I find the tax rate $\tau_{t}$ which follows a given stationary history:

$$
\tau_{t}=\frac{\overline{\theta^{2}}-\theta^{m^{2}}+\frac{\alpha(1-\tau)^{2}\left(\overline{\theta^{2}}-\theta^{m^{2}}\right)}{1-\alpha(1-\tau)}}{2 \bar{\theta}^{2}-\theta^{m^{2}}} .
$$

This expression is decreasing in $\tau$, hence there is a unique fixed point. ${ }^{16}$ Redistribution is driven by the difference between mean and median wealth and this is the case in every period. The dynamic implication is that present redistribution depends on the history of past redistributive outcomes and more precisely the tax rate declines over time. $^{17}$

\section{Random Abilities across Generations}

I now explore the case of random abilities. As I have done in the previous section with exogenous voting I assume that abilities are drawn at random for each generation and that $\theta_{t}^{i^{2}}=\bar{\theta}^{2}+\varepsilon_{t}^{i}$, where $\varepsilon_{t}^{i}$ is i.i.d. across $i$ and $t$ with mean $=0$ and variance equal to $\sigma^{2}$. Considering a stationary history $\tau_{s}=\tau$ for all generations $s \leq t-1$, the law of motion (8) implies that the mean bequest converges to expression (10) and that the variance is equal to expression (12).

In general, it is complicated to identify the median voter at time $t$ because this depends on both the distributions of $k$ and $\theta$. To see this, take expression (22) and re-

express it as $\tau_{t}^{i}\left(k_{t}^{i}, \theta_{t}^{i}\right)=\frac{\bar{\theta}^{2}-\theta^{i 2}+b\left(k_{t}^{i}-\bar{k}_{t}\right)}{2 \bar{\theta}^{2}-\theta^{i 2}}$. This shows that the distribution of $\tau_{t}^{i}$ is given by the ratio of two distributions, respectively the numerator and the denominator of expression (22). Take for example the case in which $\varepsilon_{t}^{i}$ is normally distributed and each generation starts with no endowment at time $0\left(k_{0}^{i}=0\right.$ for all $\left.i\right)$. In this case both $k^{i}$ and $\theta^{i}$ are normally distributed at time $t$ and the distribution of $\tau_{t}^{i}\left(k_{t}^{i}, \theta_{t}^{i}\right)=\frac{\bar{\theta}^{2}-\theta^{i 2}+b\left(k_{t}^{i}-\bar{k}_{t}\right)}{2 \theta^{2}-\theta^{i 2}}$, is given by the ratio of two normal distributions. It is quite complicated to identify such ratio distribution where the two normal variables have different means and to find the median. ${ }^{18}$ This difficulty to deal with dynamic models of voting in which abilities change over time has been recognized in the literature. ${ }^{19}$ 


\section{Random Abilities across Generations and Incomplete Information}

In order to skip this technical problem it is useful to consider the case of incomplete information in which everyone expects to be of average ability $\bar{\theta}$. In this case the ideal tax rate of each individual $i$ is given by expression (22) once that $\theta^{i 2}$ and $\bar{\theta}^{2}$ are replaced by $(\bar{\theta})^{2}$, obtaining

$$
\tau^{i}\left(k^{i}, \bar{\theta}\right)=\frac{b\left(k_{t}^{i}-\bar{k}_{t}\right)}{(\bar{\theta})^{2}}
$$

Given that the distribution of the ideal tax rates only depends on the distribution of $k^{i}$ it is immediate to identify the median voter to be the voter with median endowment $k^{m}$. I consider two specific distributions as examples.

Example 1: binomial distribution of abilities. In every period each individual $i$ has ability $\theta_{L}$ with probability $\pi$ and ability $\theta_{H}$ with probability $1-\pi$ and I assume that every dynasty starts life with no endowment $\left(k_{0}^{i}=0\right.$ for all $\left.i\right)$. Given a stationary history, the distribution of $k$ will converge to a normal distribution, because the distribution of $k$ comes from repeated independent Bernuolli trials over ability realizations and the distribution of those realizations converges to a normal distribution. The fact that the distribution of $k$ converges to the normal implies that at time $t$ the median and the mean endowment coincide and therefore that $\tau_{t}=0$. Thus in the case of binomial distribution of abilities there is a unique steady-state with zero tax. This is again a case in which inequality progressively decreases. Being the steady state with zero tax, there is convergency to the same steady state as in a model without redistribution. On the other hand taxation may increase the speed of convergency. ${ }^{20}$

Example 2: Log-Normal distribution of abilities. I consider the case in which $\theta_{t}^{i^{2}}=\overline{\theta^{2}}+\varepsilon_{t}^{i}$, with $\varepsilon_{t}^{i}$ i.i.d for all $i$ and $t$ and log-normally distributed. I also assume that in period 0 everyone starts life with no endowment, $k_{0}^{i}=0$ for all $i$. Given that agents have incomplete information about $\theta^{i}$ and believe to be of average ability $\bar{\theta}$, a stationary history is still specified by expressions (15), (17), (18) and $k$ is log-normally distributed in every period. Using the properties of the log-normal distribution, given $\bar{k}$ and $\operatorname{var}\left(k^{i}\right)$, the steady state median bequest is equal to $k^{m}=\frac{\bar{k}^{2}}{\sqrt{\bar{k}^{2}+\operatorname{var}\left(k^{i}\right)}}$. Given such stationary history, the voted tax rate at time $t$ is still given by $\tau_{t}=\frac{b\left(\bar{k}(\tau)-k^{m}(\tau)\right)}{\bar{\theta}^{2}}$. The difference $\bar{k}-k^{m}$ decreases in $\tau$, hence also in this case there is a unique fixed point. Noticing that $k^{m}$ decreases in $\operatorname{var}\left(k^{i}\right)$, therefore the greater it is the underlying inequality $\sigma$ and the greater it is the steady state level of redistribution $\tau$. I can solve for $\tau_{t}=\tau$ and characterize the steady state. Given that the distribution is skewed to the right, the steady state level of redistribution can be different from zero, for example the computations for the case of $\alpha=0.2, b=1, \bar{\theta}^{2}=1, \sigma^{2}=2$ imply that $\tau=0.17$. 


\section{Step 3: Endogenous Tax Rate and Endogenous Information.}

The previous section clarified the difficulties implied by the introduction of voting in a model with heterogenous abilities. In this section I allow for varying levels of information in the present dynamic model with bequests and I consider the level of information as an endogenous variable in the economy. The main technical problem with varying levels of information is still represented by the identification of the median voter. The individual ideal tax rate is determined by the individual value of wealth and the expected ability. A steady state with endogenous information must have the feature that the level of information is optimal. In order to check for this it is necessary to verify that there are no gains in changing the level of information. This is difficult because changing the level of information changes expectations, hence changes the distribution of ideal tax rates and such change is difficult to address. I provide a numerical examples with multiple politico economic equilibria. In this example both complete information $(\lambda=1)$ and minimum information $(\lambda=1 / 2)$ are optimal; the two equilibria have different macroeconomic features and can be interpreted as Europe-type versus American-type equilibria.

\subsection{Set-Up with Varying Information}

Each generation $i_{t}$ has ability $\theta_{L}$ with probability $\pi$ and ability $\theta_{H}$ with probability $1-\pi$, for all $i$ and $t$. I maintain the assumption that $\pi>1 / 2$. In each period $t$ each agent $i$ cannot observe her own or other agents' productivity but only receives a private signal $\sigma_{t}^{i}$ about the true value of $\theta_{t}^{i}$. Also the signal $\sigma_{t}^{i}$ is binary. If $\theta_{t}^{i}=\theta_{L}\left(\theta_{t}^{i}=\theta_{H}\right)$, $\sigma_{t}^{i}$ takes values $\sigma_{L}\left(\sigma_{H}\right)$ or $\sigma_{H}\left(\sigma_{L}\right)$, respectively with probability $\lambda_{t}$ and $1-\lambda_{t}$. In other words for each agent $i_{t}$ the signal $\sigma_{t}^{i}$ is independently distributed, it is truthful with probability $\lambda_{t}$, false with probability $1-\lambda_{t}$ and the transition matrix which takes from the true productivity to the signal is the following:

$$
T\left(\left[\begin{array}{c}
\sigma_{L} \\
\sigma_{H}
\end{array}\right] \mid\left[\theta_{L}, \theta_{H}\right]\right)=\left(\begin{array}{cc}
\lambda_{t} & 1-\lambda_{t} \\
1-\lambda_{t} & \lambda_{t}
\end{array}\right) .
$$

Agent's $i$ belief of the true value of $\theta_{t}^{i}$, conditional on the observation of the private signal $\sigma_{t}^{i}$, is obtained by the Bayes Rule. I introduce the following notation:

$$
\mu_{t}^{i} \equiv \operatorname{Pr}\left[\theta_{t}^{i}=\theta_{L} \mid \sigma_{t}^{i}\right]
$$

represents agent $i_{t}$ belief that $\theta_{t}^{i}=\theta_{L}$ conditional on the observation of signal $\sigma_{t}^{i}$. From the Bayes rule it follows that:

$$
\mu_{t_{\sigma_{L}}} \equiv\left(\mu_{t}^{i} \mid \sigma_{L}\right)=\frac{\pi \lambda_{t}}{\pi \lambda_{t}+(1-\pi)\left(1-\lambda_{t}\right)}
$$


and

$$
\mu_{t_{\sigma_{H}}} \equiv\left(\mu_{t}^{i} \mid \sigma_{H}\right)=\frac{\pi\left(1-\lambda_{t}\right)}{\pi\left(1-\lambda_{t}\right)+\lambda_{t}(1-\pi)} .
$$

The expected value of $\theta_{t}^{i}$ conditional on the observation of $\sigma_{t}^{i}$ is given by the following expression:

$$
\theta\left(\mu_{t}^{i}\right) \equiv \mu_{t}^{i} \theta_{L}+\left(1-\mu_{t}^{i}\right) \theta_{H}
$$

Given the symmetric structure of (25), I consider the interval $\lambda_{t} \in[1 / 2,1]$, for all $t$. For $\lambda_{t}=1 / 2$ the signal $\sigma_{t}^{i}$ is completely uninformative and the posterior belief is equal to the prior, i.e. $\mu_{t_{\sigma_{L}}}=\mu_{t_{\sigma_{H}}}=\pi$. Increasing $\lambda_{t}$ makes the signal progressively more informative up to the point that $\lambda_{t}=1$ and the signal is perfectly informative with $\mu_{t_{\sigma_{L}}}=1, \mu_{t_{\sigma_{H}}}=0$. The value of $\lambda$ represents the level of information in the economy and in a rather abstract way I consider it is an institutional feature and a policy variable. The ex-ante probability of observing $\sigma_{L}$ for each generation alive at $t$ is given by the following expression:

$$
p_{\sigma_{L t}} \equiv \operatorname{Pr}\left[\sigma_{t}^{i}=\sigma_{L}\right]=\lambda_{t} \pi+\left(1-\lambda_{t}\right)(1-\pi)
$$

symmetrically

$$
p_{\sigma_{H t}} \equiv \operatorname{Pr}\left[\sigma_{t}^{i}=\sigma_{H}\right]=\lambda_{t}(1-\pi)+\pi\left(1-\lambda_{t}\right)=1-p_{t_{\sigma_{L}}}
$$

represents the probability of observing $\sigma_{H}$. Over-lined variables stand for mean values for the population, hence $\bar{y}$ and $\bar{e}$ are respectively the mean, or aggregate, values of output and effort and

$$
\begin{gathered}
\bar{\theta} \equiv \pi \theta_{L}+(1-\pi) \theta_{H}, \\
\overline{\theta^{2}} \equiv \pi \theta_{L}^{2}+(1-\pi) \theta_{H}^{2},
\end{gathered}
$$

are respectively the mean values of productivity and squared productivity. The timing of the model is such that each individual $i$ who is alive at time $t$ starts life receiving a signal $\sigma_{t}^{i}$ and being aware of the level of precision $\lambda_{t}$. The game proceeds as in the previous sections: agents vote on tax, exert effort, receive net wealth, consume and leave bequests. The last action of the agents alive at $t$ is to collectively decide the level of information for the offspring, namely $\lambda_{t+1}$. I assume that the future level of information $\lambda_{t+1}$ is determined by majority voting.

In the case in which abilities are random in each period $t$ I would face the technical difficulties implied by the determination of the median voter which I explained in section 4. In order to keep the model tractable I restrict the analysis to the case of persistent abilities and I assume that there are only two dynasties: the low ability dynasty with ability $\theta_{L}$ in each period $t$ and the high dynasty with ability $\theta_{H}$ in each period $t$, respectively a fraction $\pi$ and $1-\pi$ of the population. Nevertheless in the case of incomplete information, agents do not learn about the persistence of abilities and 
they "falsely" believe their prior: namely that in each period $t$ abilities are i.i.d across agents and agents are of type $\theta_{L}$ with probability $\pi$ and of type $\theta_{H}$ with probability $1-\pi$ in each period $t$. On top of this prior, agents receive a signal about their ability, which will give them perfect information only in the case of $\lambda=1$.

\subsection{Politico Economic Equilibrium with Perfect Information}

Given a stationary history $s \leq t-1$ such that the voted tax rate is always $\tau_{s}=\tau$ and the voted information is always full information $\lambda_{s+1}=1$, I describe a steady state such that at time $t$, the voted tax rate is still $\tau_{t}=\tau$ and the voted information is still $\lambda_{t+1}=1$. Given a stationary history $\tau_{s}=\tau$ and $\lambda_{s+1}=1$ for $s \leq t-1$, at time $t$ the bequest for dynasty $L(H)$ converges to the steady state value $k_{L}\left(k_{H}\right)$ given by (9) and the mean bequest converges to the steady state value (10). Given the history with persistent abilities and perfect information, at time $t$ there are two groups of voters, respectively with preferred tax rates $\tau\left(k_{L}, \theta_{L}\right)$ and $\tau\left(k_{H}, \theta_{H}\right)$ given by expression (22). The prevailing tax rate at time $t$ is $\tau_{t}=\tau\left(k_{L}, \theta_{L}\right)$, because $\pi>1 / 2$ implies that the majority of the population belongs to the dynasty with low ability and low endowment. In a steady state it must be the case that $\tau_{t}=\tau$ and that the bequest that agents with endowments $k_{L}\left(k_{H}\right)$ leave for the $t+1$ offsprings is still $k_{L}\left(k_{H}\right)$.

\subsection{Politico Economic Equilibrium with Incomplete Information}

Given a stationary history $s \leq t-1$ such that the voted tax rate is always $\tau_{s}=\tau$ and the voted level of information is always the minimum $\lambda_{s+1}=1 / 2$, I describe a steady state such that at time $t$, the voted tax rate is still $\tau_{t}=\tau$ and the voted information is still $\lambda_{t+1}=1 / 2$. Given a stationary history $\tau_{s}=\tau$ and $\lambda_{s+1}=1 / 2$ for $s \leq t-1$, at time $t$ the bequest for dynasty $L(H)$ converges to the steady state value $k_{L}\left(k_{H}\right)$ given by (16) and the mean bequest converges to the steady state value (17). Given the history with persistent abilities and perfect information, at time $t$ there are two groups of voters, respectively with preferred tax rates $\tau\left(k_{L}, \bar{\theta}\right)$ and $\tau\left(k_{H}, \bar{\theta}\right)$ given by expression (24). The prevailing tax rate at time $t$ is $\tau_{t}=\tau\left(k_{L}, \bar{\theta}\right)$, because $\pi>1 / 2$ implies that the majority of the population belongs to the dynasty with low ability and low endowment. In a steady state it must be the case that $\tau_{t}=\tau$ and that the bequest that agents with endowments $k_{L}\left(k_{H}\right)$ leave for the $t+1$ offsprings is still $k_{L}\left(k_{H}\right)$.

\subsection{Solution of the Individual Problem}

In addition to leaving bequests, at time $t$ agents decide by majority voting the future information $\lambda_{t+1}$. Each agent $i_{t}$ votes on $\lambda_{t+1}$ in order to maximize the utility of the offspring $i_{t+1}$. In order to find the utility of an offspring as a function of $\lambda_{t+1}$ it is necessary to solve backwards the choices of effort and voting of the agents alive at $t+1$. Conditional on the signal, the expected utility that the generations who are alive 
at time $t$ maximize when they vote and exert effort is the following:

$$
\begin{aligned}
& E\left[u_{t}^{i} \mid \sigma_{t}^{i}\right]= \\
& E\left[\left(1-\tau_{t}\right)\left(k_{t}^{i}+e_{t}^{i} \theta_{t}^{i}\right)+\tau_{t}\left(\bar{k}_{t}+\overline{e_{t} \theta_{t}}\right)-b\left(e_{t}^{i}\right)^{2} / 2 \mid \sigma_{t}^{i}\right]
\end{aligned}
$$

Solving the sufficient first order condition, the optimal level of effort exerted by individual $i$ is

$$
e_{t}^{i}=\left(1-\tau_{t}\right) \theta\left(\mu_{t}^{i}\right) / b
$$

By backward induction, I can plug (33) into (32) and find the objective function that $i$ maximizes when voting for the tax rate. In order to do this, it is useful to specify the individual $i$ expectation of the output from effort:

$$
E\left[e_{t}^{i} \theta_{t}^{i} \mid \sigma_{t}^{i}\right]=\left(1-\tau_{t}\right)\left(\theta\left(\mu_{t}\right)\right)^{2} / b
$$

and of squared effort

$$
E\left[\left(e_{t}^{i}\right)^{2} \mid \sigma_{t}^{i}\right]=\left(e_{t}^{i}\right)^{2}=\left(\frac{1-\tau_{t}}{a}\right)^{2} \theta\left(\mu_{t}\right)^{2}
$$

In computing the mean (aggregate) product of effort $\overline{e \theta}$, each agent $i$ knows that that a fraction $\pi(1-\pi)$ of the agents have productivity $\theta_{L}\left(\theta_{H}\right)$ and that among those a fraction $\lambda$ chooses the optimal effort after the observation of $\sigma_{L}\left(\sigma_{H}\right)$, whereas a fraction $1-\lambda$ chooses the optimal effort after the observation of $\sigma_{H}\left(\sigma_{L}\right)$. Therefore the individual expectation of the aggregate output from effort is given by the following expression:

$$
E\left[\overline{e_{t} \theta_{t}} \mid \sigma_{t}^{i}\right]=\left(1-\tau_{t}\right) \Gamma / b
$$

where I define

$$
\begin{aligned}
\Gamma \equiv \pi \theta_{L}\left(\lambda \theta\left(\mu_{\sigma_{L}}\right)+(1-\lambda) \theta\left(\mu_{\sigma_{H}}\right)\right) & + \\
& (1-\pi) \theta_{H}\left((1-\lambda) \theta\left(\mu_{\sigma_{L}}\right)+\lambda \theta\left(\mu_{\sigma_{H}}\right)\right) .
\end{aligned}
$$

Collecting $\theta\left(\mu_{\sigma_{L}}\right)$ and $\theta\left(\mu_{\sigma_{H}}\right)$ it is easy to re-write expression (37) as

$$
\Gamma=p_{\sigma_{L}} \theta\left(\mu_{\sigma_{L}}\right)^{2}+\left(1-p_{\sigma_{L}}\right) \theta\left(\mu_{\sigma_{H}}\right)^{2}
$$

The term $\Gamma$ is the expression for aggregate output from effort, net of the distortive effect of redistribution on effort. Plugging (34), (35) and (37) into (32), I obtain an indirect form of (32) as a function of $\tau_{t}$ :

$$
u_{t}^{i}\left(\tau_{t}, \mu_{t}^{i}\right)=\tau_{t}\left(k_{t}^{i}-\bar{k}_{t}\right)+\left(1-\tau_{t}\right)^{2} \theta\left(\mu_{t}^{i}\right)^{2} / b+\tau_{t}\left(1-\tau_{t}\right) \Gamma / b-\left(1-\tau_{t}\right)^{2} \theta\left(\mu_{t}^{i}\right)^{2} / 2 b
$$


This is the object that voter $i_{t}$ maximizes voting over the tax rate $\tau_{t}$. Assuming for the moment that the second derivative of the obtained indirect utility in $\tau$ is strictly negative, the first order condition gives the ideal tax rate of voter $i$ :

$$
\tau\left(k_{t}^{i}, \mu_{t}^{i}\right)=1-\frac{1+\frac{b\left(k_{t}^{i}-\bar{k}_{t}\right)}{\Gamma}}{2-\frac{\theta\left(\mu_{t}^{i}\right)^{2}}{\Gamma}} .
$$

The numerator of (40) shows that the gains from redistribution are traded off the distortive effect of redistribution and the denominator of (40) shows how the subjective prospects of upward mobility reduce the desired tax rate. ${ }^{21}$

Proposition 2. The individual preferences for taxation are single peaked and the individual ideal tax rate is given by expression (40).

Proof. The second derivative of the objective function in problem (39) is given by the following expression: $\frac{d^{2} u_{1}^{i}}{d \tau}=\frac{-2 \Gamma+\theta(\mu)^{2}}{b}$. The condition stated by Assumption 1 is sufficient for this expression to be strictly negative as the maximum value that $\theta(\mu)^{2}$ can take is $\theta_{H}^{2}$ and the minimum value that $2 \Gamma$ can take is $2 \theta_{L}^{2}$.

Proposition 2 shows that preferences over the tax rate are single peaked and therefore the median voter theorem applies. Plugging (33) into (32) and taking expectations conditional on the information at time $t$, I obtain generation $i_{t}$ expectation of generation $i_{t+1}$ utility:

$$
E_{t}^{i}\left[u_{t+1}^{i}\right]=\tau_{t+1}\left(\bar{k}-k^{i}\right)+\left(1-\left(\tau_{t+1}\right)^{2}\right) \Gamma_{t+1} / 2 b .
$$

I model the collective choice of $\lambda_{t+1}$ as a choice by majority voting. Given that the agents with $k_{L}$ are the majority, their choice of $\lambda_{t+1}$ will determine the prevailing one. Therefore for a specific level of information $\lambda^{\prime}$ to be part of an equilibrium it is necessary that $\lambda^{\prime}$ is the arg $\max \left\{\tau_{t+1}\left(\bar{k}-k_{L}\right)+\left(1-\left(\tau_{t+1}\right)^{2}\right) \Gamma / 2 b\right.$. $\}$ In order to check for this it is necessary to know how $\tau_{t+1}$ changes in $\lambda$. At time $t+1$ there are four groups of voters, respectively with preferred tax rates $\tau\left(k_{L}, \theta_{\sigma_{L}}\right), \tau\left(k_{H}, \theta_{\sigma_{L}}\right)$, $\tau\left(k_{L}, \theta_{\sigma_{H}}\right), \tau\left(k_{H}, \theta_{\sigma_{H}}\right)$. I claim that in this case of $\pi>1 / 2$ the prevailing tax rate under majority voting is either $\tau\left(k_{L}, \theta_{\sigma_{L}}\right)$, or the greater between $\tau\left(k_{H}, \theta_{\sigma_{L}}\right)$ and $\tau\left(k_{L}, \theta_{\sigma_{H}}\right)$, depending on the value of $\lambda$. This claim can be easily proved. The fraction of agents who prefer $\tau\left(k_{L}, \theta_{\sigma_{L}}\right)$ is equal to $\pi p_{\sigma_{L}}$, where $p_{\sigma_{L}}$ is given by (30). When $\lambda=1$ the fraction $\pi$ of agents with endowment $k_{L}$ knows to be of type $\theta_{L}$. In the case in which $\pi>1 / 2$, this implies that they are the majority group and impose their favorite tax rate. Decreasing $\lambda$ implies that agents can have two types of beliefs, namely $\theta_{\sigma_{L}}$ and $\theta_{\sigma_{H}}$, and there are four group of voters, respectively with preferred tax rates $\tau\left(k_{L}, \theta_{\sigma_{L}}\right), \tau\left(k_{H}, \theta_{\sigma_{L}}\right), \tau\left(k_{L}, \theta_{\sigma_{H}}\right), \tau\left(k_{H}, \theta_{\sigma_{H}}\right)$. Decreasing $\lambda$ implies that $\pi p_{\sigma_{L}}$ decreases. There is a value $\lambda^{*} \in(1 / 2,1)$ such that $\pi p_{\sigma_{L}}=1 / 2$, namely $\lambda^{*}=\frac{-2 \pi+2 \pi^{2}+1}{2 \pi(2 \pi-1)}$. 
For $\lambda \in\left[1 / 2, \lambda^{*}\right)$ it happens that the group which prefers $\tau\left(k_{L}, \theta_{\sigma_{L}}\right)$ is not the majority group, and the pivotal group will be either $\tau\left(k_{H}, \theta_{\sigma_{L}}\right)$ or $\tau\left(k_{L}, \theta_{\sigma_{H}}\right)$ depending on which is the greater tax rate of the two. This is because in the case in which $\tau\left(k_{H}, \theta_{\sigma_{L}}\right)$ $\geq \tau\left(k_{L}, \theta_{\sigma_{H}}\right)$, then it is the case that the total ranking of tax rates is $\tau\left(k_{L}, \theta_{\sigma_{L}}\right)>$ $\tau\left(k_{H}, \theta_{\sigma_{L}}\right) \geq \tau\left(k_{L}, \theta_{\sigma_{H}}\right)$ and the fact that the fraction $p_{\sigma_{L}}$ of agents with belief $\theta_{\sigma_{L}}$ is greater than $1 / 2$ (this because $\pi>1 / 2$ and $\lambda>1 / 2$ ) implies that the median voter must belong to the group with $\tau\left(k_{H}, \theta_{\sigma_{L}}\right)$. Otherwise in the case in which $\tau\left(k_{L}, \theta_{\sigma_{H}}\right)$ $\geq \tau\left(k_{H}, \theta_{\sigma_{L}}\right)$, it is the case that the total ranking of tax rates is $\tau\left(k_{L}, \theta_{\sigma_{L}}\right)>\tau\left(k_{L}, \theta_{\sigma_{H}}\right)$ $\geq \tau\left(k_{H}, \theta_{\sigma_{L}}\right)$ and the fact that the fraction of agents with $k_{L}$ is greater than $1 / 2$ (this because $\pi>1 / 2)$ implies that the median voter must belong to the group with $\tau\left(k_{L}, \theta_{\sigma_{H}}\right)$.

\subsection{Example of Multiple Politico-Economic Equilibria}

I consider the following numerical example: $\theta_{L}=1, \theta_{H}=1.5, \pi=0.7, \alpha=0.2, b=1$. I first consider the case of complete information $\lambda=1$. Evaluating $k_{L}, \bar{k}$ and $\tau_{t}$ for the given values and solving for $\tau_{t}=\tau$ with I obtain that $\tau=0.243$. I plug this value back into expressions (9) and (10) and I find the steady state values $k_{L}=0.209$ and $\bar{k}=0.26$. The majority of the agents at time $t$ have endowment $k_{L}$. Under majority voting, they determine $\lambda_{t+1}$ in order to maximize the expected welfare of their offspring:

$$
u_{L}=\tau_{t+1}\left(\bar{k}-k_{L}\right)+\left(1-\left(\tau_{t+1}\right)^{2}\right) \Gamma / 2 .
$$

If $\pi p_{\sigma_{L}} \geq 1 / 2$ then the prevailing tax rate at $t+1$ is $\tau=\tau\left(k_{L}, \theta\left(\mu_{\sigma_{L}}\right)\right)$. Given $\pi=$ 0.7 and using the definition of $p_{\sigma_{L t+1}}=\pi \lambda_{t+1}+(1-\pi)\left(1-\lambda_{t+1}\right), \pi p_{\sigma_{L t+1}} \geq 1 / 2$ for $\lambda_{t+1} \geq 1.03$, therefore it is never the case that $\tau\left(k_{L}, \theta\left(\mu_{\sigma_{L}}\right)\right)$ is the pivotal. The prevailing tax rate is the greater one between $\tau\left(k_{L}, \theta\left(\mu_{\sigma_{H}}\right)\right)$ and $\tau\left(k_{H}, \theta\left(\mu_{\sigma_{L}}\right)\right)$. I plot both those two tax rates, respectively in figure 1 and 2 .

Computations show that they equal each other for 0.584 . Hence in the considered interval $\tau\left(k_{L}, \mu_{\sigma_{H}}\right)$ is greater and it is the prevailing tax rate. It is also important to notice that $\tau\left(k_{L}, \mu_{\sigma_{H}}\right) \leq 0$, for $\lambda \geq 0.55$ and that $\tau\left(k_{H}, \mu_{\sigma_{L}}\right) \leq 0$, for $\lambda \leq 0.63$. Therefore I consider $\tau=0$ in the interval $\lambda \in[0.55,0.63]$. I plot the objective function (42) in figure 3 , where $\tau_{t+1}=\tau\left(k_{L}, \mu_{\sigma_{H}}\right)$ for $\lambda_{t+1} \in[0.5,0.55], \tau_{t+1}=0$ for $\lambda_{t+1} \in$ $[0.55,0.63], \tau_{t+1}=\tau\left(k_{H}, \mu_{\sigma_{L}}\right)$ for $\lambda_{t+1} \in[0.63,1] . \lambda_{t+1}=1$ is a global maximum.

I now consider the case of completely uninformative signals $\lambda=1 / 2$. Given a stationary history $s \leq t-1$ such that the voted tax rate is $\tau_{s}=\tau$ and the voted information is the minimum information $\lambda_{s+1}=1 / 2$, I describe a steady state such that at time $t$, the voted tax rate is still $\tau_{t}=\tau$ and the voted information is still $\lambda_{t+1}=1 / 2$. Given a stationary history $\tau_{s}=\tau$ and $\lambda_{s+1}=1 / 2$ for $s \leq t-1$, at time $t$ the bequest for dynasty $L(H)$ converges to the steady state value $k_{L}\left(k_{H}\right)$ given by (16) and the mean bequest converges to the steady state value (17). Given the history with persistent abilities and minimum information, at time $t$ there are two groups of voters, respectively with preferred tax rates $\tau\left(k_{L}, \bar{\theta}\right)$ and $\tau\left(k_{H}, \bar{\theta}\right)$ given by expression (22). The prevailing tax rate 


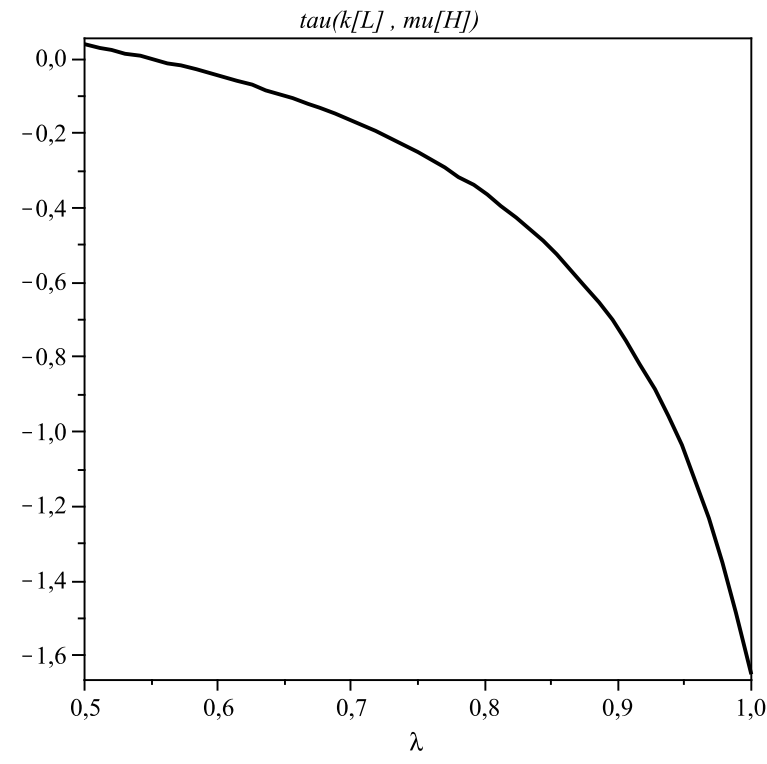

Figure 1: $\tau\left(k_{L}, \mu_{\sigma_{H}}\right)$ for $\pi=0.7, \theta_{L}=1, \theta_{H}=1.5, b=1, \alpha=0.2$.

at time $t$ is $\tau_{t}=\tau\left(k_{L}, \bar{\theta}\right)$, because $\pi>1 / 2$ implies that the majority of the population belongs to the dynasty with low ability and low endowment. In a steady state it must be the case that $\tau_{t}=\tau$ and that the bequest that agents with endowments $k_{L}\left(k_{H}\right)$ leave for the $t+1$ offsprings is still $k_{L}\left(k_{H}\right)$. Following the previous example for $\lambda=1 / 2$ to be part of a steady state it is necessary that $\lambda=1 / 2$ is the $\arg$ max expression (42).

I consider the same numerical example: $\theta_{L}=1, \theta_{H}=1.5, \pi=0.7, \alpha=0.2, b=1$. Evaluating $k_{L}, \bar{k}$ and $\tau_{t}$ for the given values and solving for $\tau_{t}=\tau$ with Maple I obtain $\tau=0.03$. I plug this value back into expressions (16) and (17) and I find the steady state values $k_{L}=0.28$ and $\bar{k}=0.32$. If $\pi p_{\sigma_{L t+1}} \geq 1 / 2$ then the prevailing tax rate at $t+1$ is $\tau=\tau\left(k_{L}, \theta\left(\mu_{\sigma_{L}}\right)\right)$. Given $\pi=0.7$ and using the definition of $p_{\sigma_{L t+1}}=$ $\pi \lambda_{t+1}+(1-\pi)\left(1-\lambda_{t+1}\right), \pi p_{\sigma_{L t+1}} \geq 1 / 2$ for $\lambda_{t+1} \geq 1.03$, therefore it is never the case that $\tau\left(k_{L}, \theta\left(\mu_{\sigma_{L}}\right)\right)$ is the pivotal. The prevailing tax rate is the greater one between $\tau\left(k_{L}, \theta\left(\mu_{\sigma_{H}}\right)\right)$ and $\tau\left(k_{H}, \theta\left(\mu_{\sigma_{L}}\right)\right)$. I plot both those two tax rates for $\lambda \in[1 / 2,1]$, respectively in figure 4 and 5 . They equal each other for 0.567 , therefore the prevailing tax rate is $\tau\left(k_{L}, \theta\left(\mu_{\sigma_{H}}\right)\right)$ for $\lambda \in[0.5,0.567]$ and $\tau\left(k_{H}, \theta\left(\mu_{\sigma_{L}}\right)\right)$ for $\lambda \in[0.567,1]$. Hence in the considered interval $\tau\left(k_{L}, \mu_{H}\right)$ prevails. It is also important to notice that $\tau\left(k_{L}, \mu_{H}\right) \leq 0$, for $\lambda \geq 0.551$, and that $\tau\left(k_{H}, \mu_{L}\right) \leq 0$, for $\lambda \geq 0.6$, therefore I consider $\tau=0$ in the interval $\lambda \in[0.567,0.6]$. I plot the objective function (42) in figure 3, where $\tau_{t+1}=\tau\left(k_{L}, \mu_{H}\right)$ for $\lambda_{t+1} \in[0.5,0.567], \tau_{t+1}=0$ for $\lambda_{t+1} \in[0.567,0.6], \tau_{t+1}=$ 


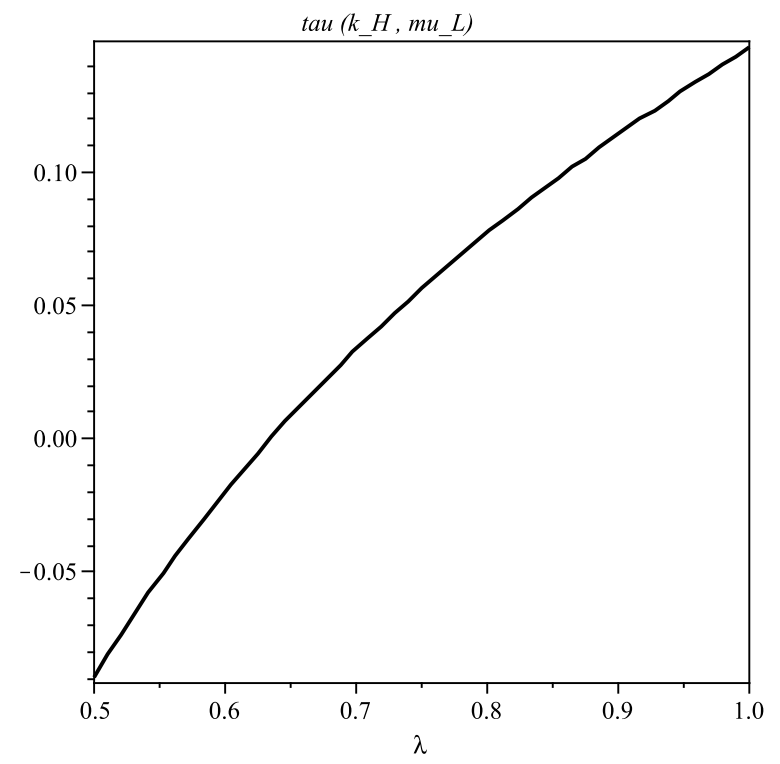

Figure 2: $\tau\left(k_{H}, \mu_{\sigma_{L}}\right)$ for $\pi=0.7, \theta_{L}=1, \theta_{H}=1.5, b=1, \alpha=0.2$.

$\tau\left(k_{H}, \mu_{L}\right)$ for $\lambda_{t+1} \in[0.6,1] . \lambda_{t+1}=1 / 2$ is a local maximum. ${ }^{22}$ The two equilibria can be further characterized in terms of the other endogenous outcomes.

\section{Values of the Endogenous Variables in the Equilibrium with Perfect Information}

Wealth of dynasty with low ability before taxes (I label it with the apex $B$ ): $w_{L}^{B}=$ $k_{L}+(1-\tau) \theta_{L}^{2}=0.965$.

Wealth of dynasty with high ability before taxes (I label it with the apex $B$ ): $w_{H}^{B}=$ $k_{H}+(1-\tau) \theta_{H}^{2}=2.080$.

Wealth of dynasty with low ability after taxes: $w_{L}=(1-\tau)\left(k_{L}+(1-\tau) \theta_{L}^{2}\right)+\tau(\bar{k}+$ $\left.(1-\tau) \bar{\theta}^{2}\right)=1.047$

Wealth of dynasty with high ability after taxes: $w_{H}=(1-\tau)\left(k_{H}+(1-\tau) \theta_{H}^{2}\right)+\tau(\vec{k}+$ $\left.(1-\tau) \bar{\theta}^{2}\right)=1.891$.

Effort exerted by low ability individuals: $e_{L}=(1-\tau) \theta_{L}=0.757$.

Effort exerted by high ability individuals: $e_{H}=(1-\tau) \theta_{H}=1.135$.

Aggregate effort: $\bar{e}=(1-\tau) \bar{\theta}=0.870$.

Aggregate output: $\bar{y}=\bar{k}+(1-\tau) \bar{\theta}^{2}=1.300$. 


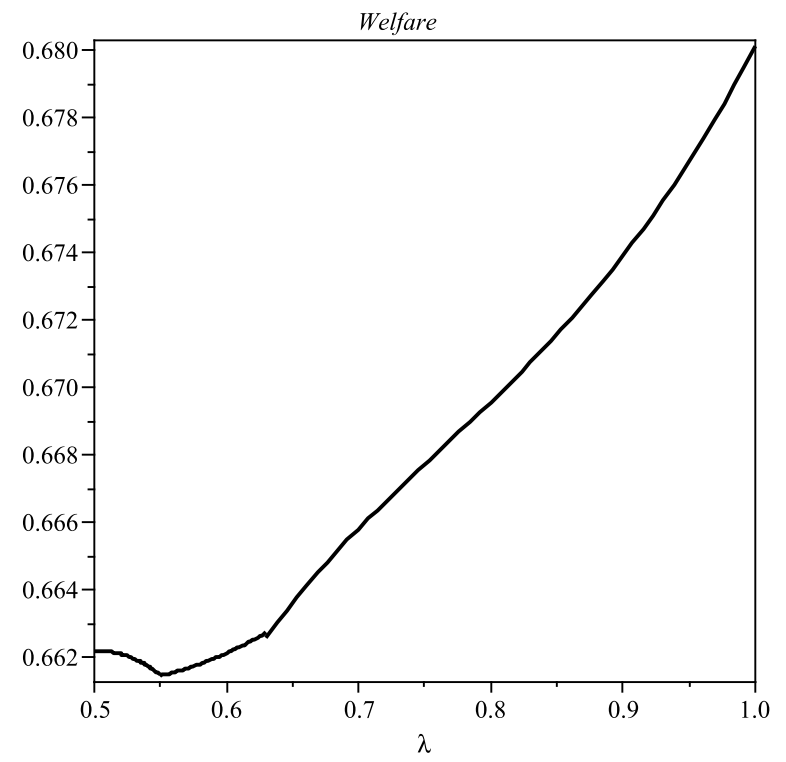

Figure 3: Objective function (42) for $\pi=0.7, \theta_{L}=1, \theta_{H}=1.5, b=1, \alpha=0.2$.

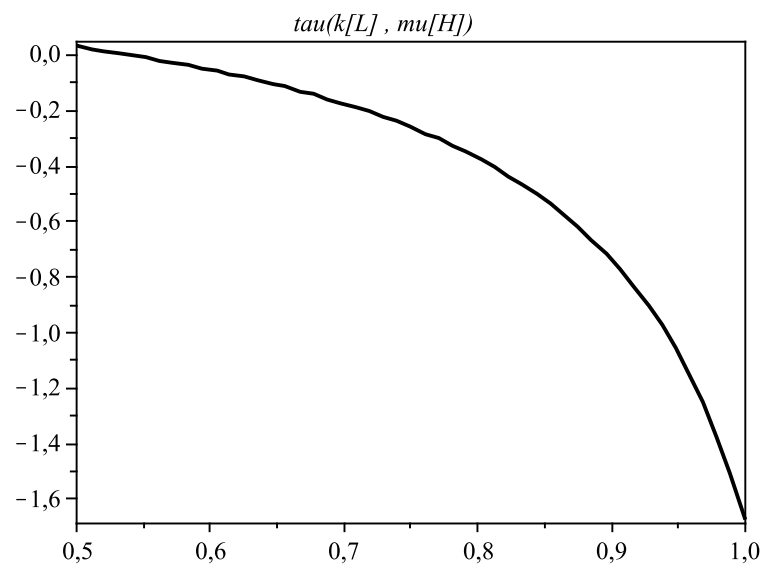

Figure 4: $\tau\left(k_{L}, \mu_{\sigma_{H}}\right)$ for $\pi=0.7, \theta_{L}=1, \theta_{H}=1.5, b=1, \alpha=0.2$.

Values of the Endogenous Variables in the Equilibrium with Perfect Information 


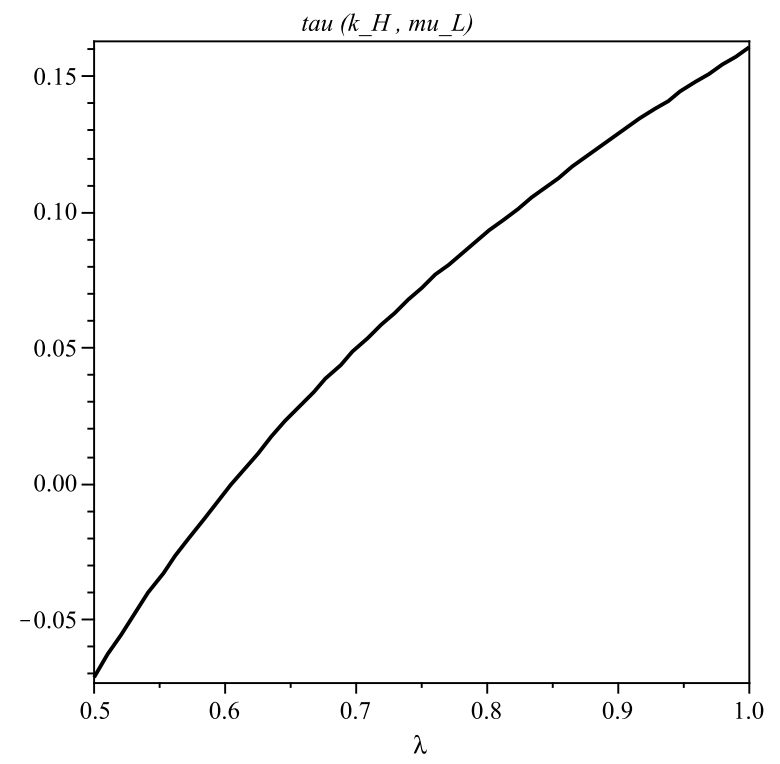

Figure 5: $\tau\left(k_{H}, \mu_{\sigma_{L}}\right)$ for $\pi=0.7, \theta_{L}=1, \theta_{H}=1.5, b=1, \alpha=0.2$.

Wealth of dynasty with low ability before taxes (I label it with the apex $B$ ): $w_{L}^{B}=$ $k_{L}+(1-\tau) \theta_{L} \bar{\theta}=1.395$.

Wealth of dynasty with high ability before taxes (I label it with the apex $B$ ): $w_{H}^{B}=$ $k_{H}+(1-\tau) \theta_{H} \bar{\theta}=2.083$.

Wealth of dynasty with low ability after taxes: $w_{L}=(1-\tau)\left(k_{L}+(1-\tau) \theta_{L} \bar{\theta}\right)+\tau(\bar{k}+$ $\left.(1-\tau) \bar{\theta}^{2}\right)=1.401$.

Wealth of dynasty with high ability after taxes: $w_{H}=(1-\tau)\left(k_{H}+(1-\tau) \theta_{H} \bar{\theta}\right)+$ $\tau\left(\bar{k}+(1-\tau) \bar{\theta}^{2}\right)=2.069$.

Effort exerted by both low and high ability individuals: $e_{L}=e_{H}=\bar{e}=(1-\tau) \bar{\theta}=$ 1.115 .

Aggregate output: $\bar{y}=\bar{k}+(1-\tau) \bar{\theta}^{2}=1.603$.

\section{Interpretation of the Result of Multiple Equilibria}

The numerical example shows the existence of two equilibria, respectively with complete $(\lambda=1)$ and with minimum $(\lambda=1 / 2)$ information. In this numerical example the multiplicity arises because $\lambda=1$ happens to be a global maximum and $\lambda=1 / 2$ is a local maximum. ${ }^{23}$ In this numerical example, the non-monotonicity of the objective function with respect to the level of information, which implies the possibility that $\lambda=1 / 2$ is a local maximum, is driven by the change in the identity of the median 


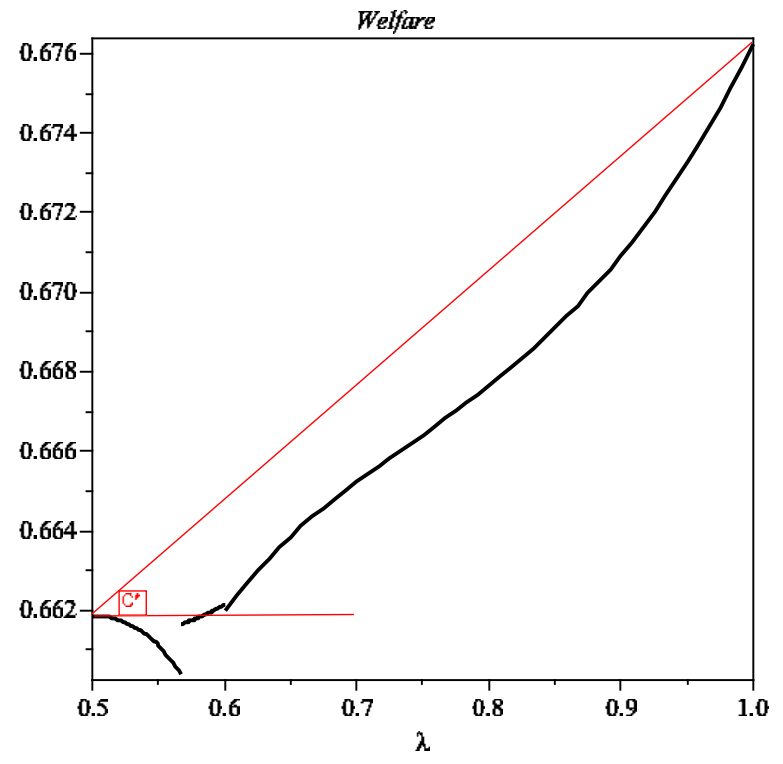

Figure 6: Objective function (42) for $\pi=0.7, \theta_{L}=1, \theta_{H}=1.5, b=1, \alpha=0.2$.

voter. In the interval in which the median voter is the group with preferred tax rate $\tau\left(k_{L}, \mu_{H}\right)$ the tax rate decreases in $\lambda$. As figure 6 suggests, in this interval this effect dominates the sign of the derivative of the objective function (42) and implies that in such interval the objective function decreases.

\section{Conclusion}

This paper developed a dynamic model with bequests, stochastic skills, endogenous voting and endogenous information. Given the technical issues involved, I proceeded by steps adding one element at the time. The intermediate steps showed results already known by the previous literature but offered a unified a framework. The result of the paper is to have taken a first step towards the development of a unifying framework which allows to study how beliefs about the determinants of wealth can affect the dynamics of inequality, mobility and redistribution.

Given the technical difficulties that stochastic skills imply in the determination of the median voter, I can only characterize equilibria with endogenous information for the case of persistent abilities over the life of dynasties $\left(\theta_{t}^{i}=\theta^{i}\right)$. In this case, with a numerical example, I show the possibility of equilibria with complete $(\lambda=1)$ and with minimum $(\lambda=1 / 2)$ information. 
This example essentially shows that societies with similar fundamentals can find optimal to be permanently "stuck" at different informative structures. Such different informative structures imply different steady states in terms of beliefs, redistribution, aggregate effort, aggregate output, separation of effort choices across individuals and inequality of wealth across individuals. Why, in this example, does it happen that a society remain stuck at a particular steady state? In terms of interpretations, the equilibria of the example describe a society which is characterized by a certain level of information long enough such that wealth is distributed so that for the majority group there is no ex-ante gain in changing the information structure. This happens because that particular level of information maximizes the welfare of the majority group. Given that in the uninformative equilibrium of the numerical example $\lambda=1 / 2$ is a local maximum, the interpretation of such equilibrium should be that of a society for which the welfare gain in increasing the level of information would be off-set by some implied structural costs of doing so. As explained at the end of the previous section, it is important to notice that for this to be the case, a necessary condition is that the welfare function does not increase monotonically in the level of information. ${ }^{24}$

The analysis of this paper does not generally show how the economic and redistributive outcomes of an equilibrium depend on the level of information but it is limited to numerical examples. The numerical examples show the possibility of equilibria with complete $(\lambda=1)$ and with minimum $(\lambda=1 / 2)$ information. Comparing the endogenous variables in the two equilibria of the example, the equilibrium with $\lambda=1$ is characterized by relatively higher redistribution, greater inequality before taxes, greater inequality after taxes, more separated levels of effort, lower aggregate effort and lower aggregate output. The informative (uninformative) equilibrium presents Europe-type (US-type) features in terms of redistribution, aggregate effort and output. The feature that the more informative equilibrium is characterized by higher inequality is not too surprising given that in the example the uninformative equilibrium is characterized by minimum information and levels of effort which are identical across different types of agents. One would expect such pooling equilibrium to be associated with low inequality. In principle it does not have to be the case that more informative equilibria are always associated with higher inequality. The opposite could happen if in the more informative equilibrium the higher rate of redistribution distorts effort so much that the levels of exerted effort are less separated, despite the fact that beliefs are more separated. Interestingly enough given the mentioned empirical evidence about redistribution and inequality, in such a case the driving force behind higher taxation would not be the actual level of inequality, even though this would still impact on the ideal tax rate, but the beliefs about the determinants of wealth. Moreover, in such a case, the driving force behind the fact that effort levels are less separated in the Europe-type equilibrium would be the distortive effect of taxation. ${ }^{25}$

In order to verify this possibility it is necessary to look for equilibria with interior solutions in terms of information because the extreme cases do not seem to give that result. Even in the case of persistent abilities, with interior solutions the identification 
of the median voter is problematic. Allowing for the case in which abilities evolve stochastically across generations could give interesting results about the study of mobility and inequality, because it would be possible to characterize how different levels of information impact on effort decisions and taxation and obtain interesting comparative statics. Therefore, given the implied technical issues in the identification of the median voter, it seems a promising direction for future research to investigate those issues with numerical methods.

\section{Notes}

${ }^{1}$ Tommaso Gabrieli is Assistant Professor at Henley Business School, University of Reading. This paper is a revised version of Chapter III of my Phd thesis, submitted for the degree of Doctor of Philosophy in Economics at the University of Warwick. I thank Herakles Polemarchakis and Sayantan Ghosal for invaluable guidance and motivation as my PhD supervisors. Correspondence: School of Real Estate and Planning, Whiteknights Campus, University of Reading, RG66UR, Reading, UK.

${ }^{2}$ More precisely, from incomplete to perfect information about the value of the return to effort versus the role of luck or other predetermined factors.

${ }^{3}$ Students' tracking (see for example Woessmann (2009)) or screening (see for example Bishop (1997)).

${ }^{4}$ See for example the discussion of Piketty (1995).

${ }^{5}$ See for example the discussion of Alesina and Glaeser (2004).

${ }^{6}$ A more extensive review can be found in Gabrieli (2009).

${ }^{7}$ See Piketty (1998) for an extensive review of the literature on bequests and intergenerational mobility.

${ }^{8}$ See for example Benabou (1996).

${ }^{9}$ The paper of Alesina and Angeletos (2005) contains a dynamic version of the main model. In this dynamic version skills are fixed and permanently different across generations. For this reason the model can show how beliefs can persist and societies can remain stuck in different equilibria as a result of different initial conditions, but the model cannot give insights about mobility. Also the model of Piketty (1995) is dynamic, but does not allow for intergenerational transfers. The dynamic aspect only concerns the learning about the determinants of wealth. The model can give insights about the beliefs on mobility but not about the actual mobility process.

${ }^{10}$ The process is convergent as the coefficient of $k_{t}^{i}$ is $\alpha(1-\tau)<1$ and the rest of expression (8) is constant.

${ }^{11}$ This is due to the linear production function and it is different from other models with concave production functions, where redistribution can improve the efficiency of the inputs' allocations and hence increase output, see for example Galor and Zeira (1993) and Benabou (2000).

${ }^{12}$ In order to prove convergency, it is enough to cite the result of Hellwig (1980) which applies to Markov processes of this type.

${ }^{13}$ It is easy to compute that expression (12) monotonically decreases in $\tau$ : $d\left(\operatorname{var}\left(k^{i}\right)\right) / d \tau=\frac{\alpha^{2} \sigma^{2}}{b^{2}}\left[\frac{-4(1-\tau)^{3}\left(1-\alpha^{2}(1-\tau)^{2}\right)-2 \alpha^{2}(1-\tau)^{5}}{\left(1-\alpha^{2}(1-\tau)^{2}\right)^{2}}\right]<0$, given $\tau<1$

${ }^{14}$ A detailed analysis of the results of Stiglitz (1969) in the context of intergenerational transmission of wealth is contained in the review of Piketty (1998). Piketty (1998) explains that, as shown by Bourguignon (1981), in the case of convex bequest function the result about convergency does not generally hold. Other causes that can imply non convergency are different fertility behaviours across households and credit market imperfections. He also discusses the case of random abilities.

${ }^{15}$ Information improves the efficiency of effort allocations and increases output, see Gabrieli (2010) for a discussion.

${ }^{16}$ This result of a unique steady state is found also by Alesina and Angeletos (2005) and it is the standard result of Meltzer and Richard (1981) in a dynamic context. 


\footnotetext{
${ }^{17}$ The same result is also found by Bertocchi (2011) in a model which specifically analyzes the evolution of bequest taxation over time.

${ }^{18} \mathrm{~A}$ technical analysis of the properties of this type of ratio distribution is done by Hinkley (1969).

${ }^{19}$ To my knowledge, the only analytical dynamic model of voting in which abilities change over time is the one of Persson and Tabellini. (1991), but in this paper there is no identification of the median voter in a steady state. In the published version of the paper (Persson and Tabellini (1994)) it is only considered the case of persistent abilities. Alesina and Rodrik (1994), Das and Ghate (2004), Hassler, Rodriguez-Mora, Storesletten, and Zilibotti (2003), Saint-Paul (2001), Bertocchi (2007) only consider the case of persistent abilities.

${ }^{20}$ Also this point has been discussed by Stiglitz (1969).

${ }^{21}$ The term $\frac{\theta\left(\mu^{i}\right)^{2}}{\Gamma}$ represents subjective prospects of upward mobility as it is equal to the the ratio of individual output (34) over aggregate output (36), noticing that the term $\frac{1-\tau}{a}$ gets canceled out.

${ }^{22}$ Therefore with a linear cost of changing information $C\left(\lambda^{\prime}-1 / 2\right)$ greater than the slope of the line connecting the welfare function at $\lambda=1 / 2$ and at $\lambda=1$ it is an equilibrium, as shown in figure 6 .

${ }^{23}$ Therefore for $\lambda=1 / 2$ to be optimal it is necessary to introduce the additional assumption that there is a cost of increasing the level of information which is large enough. It is worthy to mention that in the model of Benabou and Tirole (2006) for multiple equilibria to exist the same assumption is necessary.

${ }^{24}$ More specifically, it is a case in which increasing information implies that the median voter sets a level of taxation which is too high in terms of ex-ante aggregate welfare. See Gabrieli (2010) for a more extensive discussion of this property.

${ }^{25}$ This point seems to have some robust empirical support, see for example Prescott (2004).
}

\section{References}

Aghion, P., And P. Bolton (1997): "A Theory of Trickle-Down Growth and Development," Review of Economic Studies, 64(2), 151-72.

Alesina, A., And G.-M. Angeletos (2005): "Fairness and Redistribution: US versus Europe," American Economic Review, 95, 960-980.

Alesina, A., And E. Glaeser (2004): Fighting Poverty in the US and Europe: A World of Difference. Oxford University Press, Oxford, UK.

Alesina, A., And D. Rodrik (1994): "Distributive Politics and Economic Growth," The Quarterly Journal of Economics, 109(2), 465-490.

Atkinson, A. (1980): "Inheritance and the Redistribution of Wealth," in Public Policy and the Tax System: Essays in honour of James Meade, ed. by G. Hughes, and G. Heal. London: Allen and Unwin.

Banerjee, A. V., And A. F. Newman (1993): “Occupational Choice and the Process of Development," Journal of Political Economy, 101(2), 274-98.

Becker, G. S., And N. Tomes (1979): "An Equilibrium Theory of the Distribution of Income and Intergenerational Mobility,” Journal of Political Economy, 87(6), 1153-89. 
(1986): "Human Capital and the Rise and Fall of Families," Journal of Labor Economics, 4(3), 1-39.

Benabou, R. (1996): "Inequality and Growth," in NBER Macroeconomics Annual 1996, ed. by B. S. Bernanke, and J. Rotemberg. Cambridge, MA: MIT Press.

(2000): "Unequal Societies: Income Distribution and the Social Contract," American Economic Review, 90(1), 96-129.

Benabou, R., and J. TiRole (2006): "Belief in a Just World and Redistributive Politics," Quarterly Journal of Economics, 121, 699-746.

Bertocchi, G. (2007): "The Vanishing Bequest Tax: The Comparative Evolution of Bequest Taxation in Historical Perspective," IZA Discussion Papers 2578, Institute for the Study of Labor (IZA).

(2011): "The Vanishing Bequest Tax: The Comparative Evolution Of Bequest Taxation In Historical Perspective," Economics and Politics, 23(1), 107-131.

Bishop, J. H. (1997): "The Effect of National Standards and Curriculum-Based Exams on Achievement," American Economic Review, 87(2), 260-264.

Bourguignon, F. (1981): "Pareto Superiority of Unegalitarian Equilibria in Stiglitz' Model of Wealth Distribution with Convex Saving Function," Econometrica, 49(6), 1469-1475.

DAS, S., AND C. Ghate (2004): "Endogenous Distribution, Politics, and the GrowthEquity Tradeoff," Contributions to Macroeconomics, 4(1), 1199-1199.

Gabrieli, T. (2009): "Redistributive Politics under Optimally Incomplete Information," Ph.D. thesis, University of Warwick, UK.

(2010): "Diverse Societal Beliefs and Redistributive Policies, but Equal Welfare: The Trade-off Effect of Information," City University Economics Discussion Papers 10/04, Department of Economics, City University, London.

Galor, O., AND J. ZeIRA (1993): "Income Distribution and Macroeconomics," Review of Economic Studies, 60(1), 35-52.

Hassler, J., J. V. Rodriguez-Mora, K. Storesletten, and F. Zilibotti (2003): "The Survival of the Welfare State," American Economic Review, 93(1), $87-112$.

Hellwig, M. F. (1980): "Stochastic processes of temporary equilibria : A note," Journal of Mathematical Economics, 7(3), 287-299. 
HINKLEY, D. V. (1969): “On the Ratio of Two Correlated Normal Random Variables," Biometrika, 56(3), 635-639.

Meltzer, A. H., And S. F. Richard (1981): "A Rational Theory of the Size of Government," Journal of Political Economy, 89, 914-927.

Perotti, R. (1993): "Political Equilibrium, Income Distribution, and Growth," Review of Economic Studies, 60(4), 755-76.

Persson, T., and G. TABellini. (1991): "Is Inequality Harmful for Growth? Theory and Evidence," Economics Working Papers 91-155, University of California at Berkeley.

Persson, T., And G. TABellini (1994): "Is Inequality Harmful for Growth?," The American Economic Review, 84(3), 600-621.

PiketTy, T. (1995): "Social Mobility and Redistributive Politics," Quarterly Journal of Economics, 110, 551-584.

(1997): "The Dynamics of the Wealth Distribution and the Interest Rate with Credit Rationing," RES, 64(2), 173-190.

(1998): "Theories of persistent inequality and intergenerational mobility," in Handbook of Income Distribution, ed. by A. Atkinson, and F. Bourguignon. Amsterdam: North Olland.

PRESCOTt, E. C. (2004): "Why do Americans work so much more than Europeans?," Federal Reserve Bank of Minneapolis Quarterly Review, 28(1), 2-13.

ROMER, T. (1975): "Individual welfare, majority voting, and the properties of a linear income tax," Journal of Public Economics, 4(2), 163-185.

Saint-Paul, G. (2001): "The Dynamics of Exclusion and Fiscal Conservatorism," Review of Economic Studies, 4, 275-302.

Solow, R. M. (1956): "A Contribution to the Theory of Economic Growth," The Quarterly Journal of Economics, 70(1), 65-94.

StiglitZ, J. E. (1969): "Distribution of Income and Wealth among Individuals," Econometrica, 37(3), 382-97.

Woessmann, L. (2009): "International Evidence on School Tracking: a review," Discussion paper, CESifo DICE Report 1/2009. 